\title{
Study of breast cancer incidence in patients of lymphangioleiomyomatosis
}

\author{
Olivier Nuñez ${ }^{1}$ - Antonio Román ${ }^{2} \cdot$ Simon R. Johnson ${ }^{3} \cdot$ Yoshikazu Inoue $^{4}$. \\ Masaki Hirose $^{4}$ - Álvaro Casanova ${ }^{5}$ Gorka Ruiz de Garibay ${ }^{6}$ - Carmen Herranz $^{6}$. \\ Gema Bueno-Moreno ${ }^{7,8}$ - Jacopo Boni ${ }^{6}$ - Francesca Mateo ${ }^{6} \cdot$ Anna Petit' $^{9}$. \\ Fina Climent ${ }^{9} \cdot$ Teresa Soler $^{9} \cdot$ August Vidal $^{9} \cdot$ José Vicente Sánchez-Mut $^{10}$. \\ Manel Esteller ${ }^{10,11,12}$ - José Ignacio López ${ }^{13}$ - Nadia García ${ }^{6}$ - Anna Gumà ${ }^{14}$. \\ Raúl Ortega $^{14}$ - María Jesús Plà ${ }^{15}$ - Miriam Campos ${ }^{16}$ - Emilio Ansótegui ${ }^{17}$. \\ María Molina-Molina $^{18,19}$. Claudia Valenzuela ${ }^{20}$ - Piedad Ussetti ${ }^{21}$. \\ Rosalía Laporta $^{21}$ - Julio Ancochea ${ }^{20}$ - Antoni Xaubet ${ }^{19,22} \cdot$ Marina Pollán $^{1}$. \\ Miguel Angel Pujana ${ }^{6}$
}

Received: 4 January 2016/Accepted: 27 February 2016/Published online: 7 March 2016

(C) The Author(s) 2016. This article is published with open access at Springerlink.com

\begin{abstract}
Molecular evidence has linked the pathophysiology of lymphangioleiomyomatosis (LAM) to that of metastatic breast cancer. Following on this observation, we assessed the association between LAM and subsequent breast cancer. An epidemiological study was carried out using three LAM country cohorts, from Japan, Spain, and the United Kingdom. The number of incident breast cancer
\end{abstract}

Electronic supplementary material The online version of this article (doi:10.1007/s10549-016-3737-8) contains supplementary material, which is available to authorized users.

Marina Pollán

mpollan@isciii.es

$\triangle$ Miguel Angel Pujana

mapujana@iconcologia.net

1 Cancer and Environmental Epidemiology Unit, National Center for Epidemiology, Carlos III Institute of Health, and Consortium for Biomedical Research in Epidemiology and Public Health (CIBERESP), Sinesio Delgado 6, 28029 Madrid, Spain

2 Lung Transplant Unit, Department of Pulmonology, Lymphangioleiomyomatosis Clinic, Vall d'Hebron University Hospital, 08035 Barcelona, Catalonia, Spain

3 National Centre for Lymphangioleiomyomatosis, Nottingham University Hospitals NHS Trust, Nottingham,

Nottinghamshire, UK Division of Respiratory Medicine and Respiratory Research Unit, University of Nottingham, Nottingham NG7 2UH, UK

4 National Hospital Organization Kinki-Chuo Chest Medical Center, Sakai 591-8555, Osaka, Japan

5 Department of Pneumology, Henares Hospital, 28882 Madrid, Spain cases observed in these cohorts was compared with the number expected on the basis of the country-specific incidence rates for the period 2000-2014. Immunohistochemical studies and exome sequence analysis were performed in two and one tumors, respectively. All cohorts revealed breast cancer standardized incidence ratios (SIRs) $\geq 2.25$. The combined analysis of all cases or restricted to pre-menopausal age groups revealed significantly higher incidence of breast cancer: $\mathrm{SIR}=2.81,95 \%$ confidence interval $(\mathrm{CI})=1.32-5.57, P=0.009 ;$ and $\mathrm{SIR}=4.88, \quad 95 \% \quad \mathrm{CI}=2.29-9.99, \quad P=0.0007$,

6 ProCURE, Breast Cancer and Systems Biology, Catalan Institute of Oncology (ICO), Bellvitge Institute for Biomedical Research (IDIBELL), Gran via 199, L'Hospitalet del Llobregat, 08908 Barcelona, Catalonia, Spain

7 Department of Biochemistry, Autonomous University of Madrid (UAM), Biomedical Research Institute “Alberto Sols" (Spanish National Research Council (CSIC)-UAM), Hospital La Paz Institute for Health Research (IdiPAZ), 28029 Madrid, Spain

8 MD Anderson International Foundation, 28033 Madrid, Spain

9 Department of Pathology, University Hospital of Bellvitge, IDIBELL, L'Hospitalet del Llobregat, 08908 Barcelona, Catalonia, Spain

10 Cancer Epigenetics and Biology Program, IDIBELL, L'Hospitalet del Llobregat, 08908 Barcelona, Catalonia, Spain

11 Department of Physiological Sciences II, School of Medicine, University of Barcelona, 08908 Barcelona, Catalonia, Spain

12 Catalan Institution for Research and Advanced Studies (ICREA), 08010 Barcelona, Catalonia, Spain 
respectively. Immunohistochemical analyses showed positivity for known markers of lung metastatic potential. This study suggests the existence of increased breast cancer risk among LAM patients. Prospective studies may be warranted to corroborate this result, which may be particularly relevant for pre-menopausal women with LAM.

Keywords Breast cancer - Incidence .

Lymphangioleiomyomatosis $\cdot$ mTOR $\cdot$ TSC1 $\cdot$ TSC2

\section{Introduction}

LAM is a rare neoplastic disease that appears predominantly in women of childbearing age and is characterized by cystic lung destruction $[1,2]$. LAM lesions are heterogeneous at the cellular phenotypic level but are typically characterized by the proliferation of estrogen receptor $\alpha$ $(\mathrm{ER} \alpha)$ - and progesterone receptor (PR)-positive smooth muscle-like cells with lung metastatic potential whose tissue origin remains unclear [3]. LAM cells commonly carry loss-of-function mutations in the tumor suppressor genes $T S C 1$ or $T S C 2$, and consequently, exhibit abnormal activation of the mechanistic target of rapamycin complex 1 (mTORC1) [4]. Thus, LAM can occur as an isolated

13 Cruces University Hospital, BioCruces Research Institute, University of the Basque Country, 48903 Barakaldo, Spain

14 Department of Radiology, University Hospital of Bellvitge, IDIBELL, L'Hospitalet del Llobregat, 08908 Barcelona, Catalonia, Spain

15 Breast Cancer Functional Unit, Department of Gynecology, University Hospital of Bellvitge, ICO, IDIBELL, L'Hospitalet del Llobregat, 08908 Barcelona, Catalonia, Spain

16 Breast Cancer Functional Unit, Department of Medical Oncology, ICO, IDIBELL, L'Hospitalet del Llobregat, 08908 Barcelona, Catalonia, Spain

17 Lung Transplant and Cystic Fibrosis Unit, Hospital Universitario y Politecnico La Fe, 46026 Valencia, Spain

18 Department of Pneumology, University Hospital of Bellvitge, IDIBELL, L'Hospitalet del Llobregat, 08908 Barcelona, Catalonia, Spain

19 Consortium for Biomedical Research in Respiratory Diseases (CIBERES), 28029 Madrid, Spain

20 Department of Pneumology, Instituto de Investigación Sanitaria La Princesa, Hospital La Princesa, 28006 Madrid, Spain

21 Department of Pneumology, University Hospital Clínica Puerta del Hierro, 28222 Madrid, Spain

22 Department of Pneumology, Hospital Clinic of Barcelona, August Pi Suñer Biomedical Research Institute (IDIBAPS), 08036 Barcelona, Catalonia, Spain disease (termed sporadic LAM) or in association with another rare disorder, tuberous sclerosis complex.

mTORC1 regulates a cancer metastasis transcriptional program [5]. In breast cancer, low expression of TSCl or TSC2 is associated with poor clinical outcome [6], and depletion of TSC2 expression promotes lung metastasis [7]. These observations led us to the test whether the mediators of breast cancer metastasis to lung could also play a role in LAM. Thus, we identified molecular positivity in LAM lesions for known metastasis mediators [8]. Low TSC1/2 expression in primary breast tumors was found to be associated with enhanced mTORC1 signaling and lung (but not bone) metastasis. Collectively, the clinical, pathological, and molecular similarities between LAM and breast cancer prompted us to hypothesize a higher incidence of breast cancer in LAM patients. To assess this hypothesis, we compiled the largest epidemiological LAM study to date.

\section{Methods}

\section{LAM cohorts}

The cohorts comprised LAM patients from three countries (Japan, Spain, and the United Kingdom). Patients were diagnosed by computed tomography scan. In most cases ( $>80 \%$ of patients in any cohort), diagnosis was complemented by the presence of at least one of the following findings: lymphatic complication, lung biopsy, renal angiomyolipoma, and/or TSC $[2,9,10]$. Collectively, the number of diagnoses per population was consistent with the reported prevalence of the disease in developed countries (1-9 in $10^{6}$ individuals) [10]: 108, 175, and 175 in Japan, Spain, and the United Kingdom, respectively. The ethics committee of the Hospital de Henares approved the international epidemiological study (approval number PI-753). The data from the Japan and United Kingdom cohorts (irreversibly encoded) were provided for combined analysis at the Spanish study center. Informed consent was not required for the epidemiological study (PI-753), as it was based on irreversibly encoded retrospective records; however, approved informed consent was obtained from those patients that provided tumor samples for genetic and immunohistochemical analyses. These studies were approved by the ethics committees of the Bellvitge Institute for Biomedical Research (IDIBELL; PR082/13), the Instituto de Investigación Sanitaria La Princesa (SEPAR2012), and the Hospital de Henares (PI-753). In addition, all LAM patients in the Japanese cohort provided informed consent for the comprehensive analysis of their clinical data (National Hospital Organization Kinki-Chuo Chest Medical Center, approval number 365 ). 


\section{Follow-up and breast cancer ascertainment}

In all clinical settings, LAM patients underwent regular follow-up evaluations with a periodicity of 3-12 months, depending on the country and each patient's condition. In addition, follow-up was updated via telephone, and patient conditions, including death, were recorded in each database. Given that LAM has only been monitored for a short time [9], and since breast cancer screening programs were not fully implemented in some countries until relatively recently, only patients diagnosed from 2000 were considered in this study, which corresponded to $>80 \%$ of the cases in each cohort (Supplementary Table 1). In all cases, follow-up started with LAM diagnosis and finished with the first occurrence of one of the following events: death, breast cancer diagnosis, date of last contact, or end of the study (December 31st, 2014). Breast cancer diagnosis required pathological confirmation. Since national breast cancer rates do not include in situ tumors, only invasive cases were considered.

\section{Statistical analysis}

The incidence of breast cancer in the LAM cohorts was compared with the incidence observed in the general population, using standardized incidence ratios (SIRs) [11]. This ratio corresponds to the observed versus expected number of cases, where the expected number is obtained considering age (5-year groups) and period- (2000-2004, 2005-2009, and 2010-2014) specific incidence breast cancer rates in each country. The same analysis was repeated considering pre-menopausal age groups (women younger than 50). Person-years in each stratum were calculated using the survival package (version 2.38 , R software), and SIR confidence intervals were computed under the Poisson assumption and using the exact method [12].

\section{Antibodies}

The antibodies used in this study were anti-ER $\alpha$ (\#IR151, Dako), anti-FSCN1 (\#SC-56531, Santa Cruz Biotechnology), anti-HMB-45 (\#SC-59305, Santa Cruz Biotechnology), anti ID1 (\#SC-488, Santa Cruz Biotechnology), antiPR (\#IR168, Dako), anti-phospho-Ser235-236 S6 ribosomal protein (anti-pS6; clone 91B2, Cell Signaling Technology), anti-SMA (\#A2547, Sigma-Aldrich), and antiSOX9 (\#AB5535, Millipore).

\section{Immunohistochemistry}

Immunohistochemical assays were performed using standard protocols with the EnVision (Dako) method. Each tissue and biomarker was evaluated in at least two independent assays and no substantial differences were observed. Equivalent sections were processed to include incubation with immunoglobulin controls (Sigma-Aldrich), which did not reveal staining in any case. The immunohistochemistry results were evaluated independently by at least two expert pathologists.

\section{Exome sequencing}

Breast cancer ( $>50 \%$ tumor cells) DNA was extracted from a surgical sample following standard protocols and the exome sequence analyzed by GATC Biotech. The coverage was of $>10 \times$ for at least $90 \%$ of the genome and some TSC1/2 exonic regions were targeted by Sanger sequencing to obtain full annotation. Variant mapping, alignment, calling, annotation, and filtration were performed using the genome reference hg19 (GRCh37) and the GATK modules [13].

\section{Results}

\section{Analysis of standardized incidence ratios}

The three LAM cohorts revealed breast cancer SIRs $\geq 2.25$ (Table 1, which also includes the observed and expected numbers per cohort and for the combined analysis). In fact, the United Kingdom cohort revealed a significant SIR of $3.16 ; 95 \%$ confidence interval $(C I)=1.08-8.15, P=0.039$. Thus, the global estimation of an excess of breast cancer cases was found to be significant: $\mathrm{SIR}=2.81,95 \% \mathrm{CI}=1.32-5.57, P=0.009$ (Table 1).

Since LAM is generally diagnosed in women of fertile age and an increased risk of breast cancer may be associated with earlier age of onset [14], an analysis restricted to pre-menopausal age groups (women younger than 50) was performed. A significantly higher incidence of breast cancer in the cohorts of Spain and the United Kingdom was observed, with SIRs $=4.98$ and $7.09 ; P$ values $=0.023$ and 0.003 , respectively. Moreover, the global analysis was also found to be significant: $\operatorname{SIR}=4.88, \quad 95 \%$ $\mathrm{CI}=2.29-9.99, P=0.0007$ (Table 1).

\section{Immunohistochemical features of breast tumors from LAM patients}

Approximately, two-thirds of the breast tumors that develop in pre-menopausal women in the general population are hormone receptor-positive [15]. In our study, all tumors with available pathological information $(n=8)$ were recorded as ER $\alpha$ - and PR-positive. While none presented family history of the disease, relatively uncommon 
Table 1 Number of breast cancer cases observed and expected, and SIRs in the three LAM cohorts

\begin{tabular}{|c|c|c|c|c|c|c|}
\hline \\
\hline Country & Person-years & $\begin{array}{l}\text { Observed } \\
\text { cases }(n)\end{array}$ & $\begin{array}{l}\text { Expected } \\
\text { cases }(n)\end{array}$ & SIR & $95 \%$ CI & $P$ value \\
\hline Japan & 605.02 & 1 & 0.44 & 2.25 & $0.12-12.96$ & 0.36 \\
\hline Spain & 899.01 & 3 & 1.13 & 2.64 & $0.72-7.77$ & 0.11 \\
\hline United Kingdom & 809.22 & 4 & 1.26 & 3.16 & $1.08-8.15$ & 0.039 \\
\hline Combined & 2313.26 & 8 & 2.84 & 2.81 & $1.32-5.57$ & 0.009 \\
\hline
\end{tabular}

Pre-menopausal $(<50$ years old $)$

\begin{tabular}{lllllll}
\hline Country & Person-years & $\begin{array}{l}\text { Breast cancer } \\
\text { cases/cohort }(n)\end{array}$ & $\begin{array}{l}\text { Expected } \\
\text { cases }(n)\end{array}$ & SIR & 95 \% CI & $P$ value \\
\hline Japan & 453.32 & 0 & 0.27 & - & - & - \\
Spain & 620.00 & 3 & 0.60 & 4.98 & $1.36-14.63$ & 0.023 \\
United Kingdom & 568.36 & 4 & 0.56 & 7.09 & $2.42-18.28$ & 0.003 \\
Combined & 1641.68 & 7 & 1.43 & 4.88 & $2.29-9.99$ & 0.0007 \\
\hline
\end{tabular}

clinical and histopathological features were noted. Three Spanish cases were diagnosed with multifocal breast cancer, one of them was diagnosed at age 37 with rapid progression following 8 months of rapamycin treatment for LAM [10]. Whole exome sequencing did not identify TSC1/2 mutations in a fresh tumor sample for this case; importantly, exonic TSC1/2 mutations are common but not seen in all sporadic LAM patients [16]. The tumor exome analysis did uncover a known oncogenic mutation in PIK3CA (c.3140A > G, H1047R), which was confirmed by Sanger sequencing (Supplementary Fig. 1); however, mutations in this gene are found relatively frequent in ER $\alpha$ positive breast cancer [17].

Immunohistochemical analyses of the above depicted tumor and of an additional case showed positivity for both $\mathrm{ER} \alpha$ and PR in cells with apparently different phenotypes (Fig. 1). Both cases were also positive for a canonical marker of mTORC1 activity and for the lung metastatic mediators revealed in our original study (FSCN1, ID1, and SOX9; Fig. 2). Heterogeneity was also apparent and positive cells were linked to either an epithelial or a spindle phenotype (Fig. 2). Together, the observations of multifocal ER $\alpha$-positive tumors in pre-menopausal LAM patients without family history of the disease would further suggest a link to a specific breast cancer subtype.

\section{Discussion}

In previous analyses, we tested the hypothesis that the metastatic properties of LAM cells could be further depicted using knowledge of breast cancer tropism to lung, and thus identified the expression of metastatic mediators and cancer cell stemness molecular determinants in LAM lesions [8]. Following on from this evidence, and given that the tissue of origin of LAM cells remains a subject of debate [3, 18, 19], we aimed to assess breast cancer incidence in LAM patients. The results of our study of three cohorts in different countries suggest that LAM patients, particularly those in the pre-menopausal age range, are at higher risk of developing invasive breast cancer than women from the general population. This might therefore indicate a shared cell origin and/or shared genetic risk factors between the two diseases. However, we cannot rule out that the retrospective nature of the survey and/or the regular clinical monitoring of LAM patients may have led to an over-estimation of breast cancer incidence.

Apart from brain and kidney malignancies [20], there is no previous evidence of other cancer susceptibilities in patients with tuberous sclerosis complex; indeed, among the LAM patients with breast cancer in our study, only one $(1 / 15 ; 6,7 \%)$ was diagnosed with this disease (i.e., carrier of a germline TSC2 mutation), so the others were sporadic LAM cases. Intriguingly, however, a recent study of 1,000 breast cancer patients incidentally identified a pathogenic germline TSC2 mutation [21]; therefore, further germline studies of TSC1/2 may be warranted to assess the potential link with breast cancer risk. In our study, exome analysis of a breast tumor sample did not reveal mutations in these genes, but exonic mutations are not detected in all sporadic LAM patients [16], which further suggests heterogeneity in the biology of LAM.

Hypothetically, multiple tissue or organ origins can coexist if it is considered that enhanced mTORC1 activity mediates metastatic behavior in different cancer types [5]. Nevertheless, the link between LAM and women at childbearing age $[2,10]$ suggests tissue and/or cell type specificity. Dependence on hormone signaling is a hallmark of several cell types in breast tissue [22]. In this scenario, the repeated cycles of vast cell proliferation that 
(a)

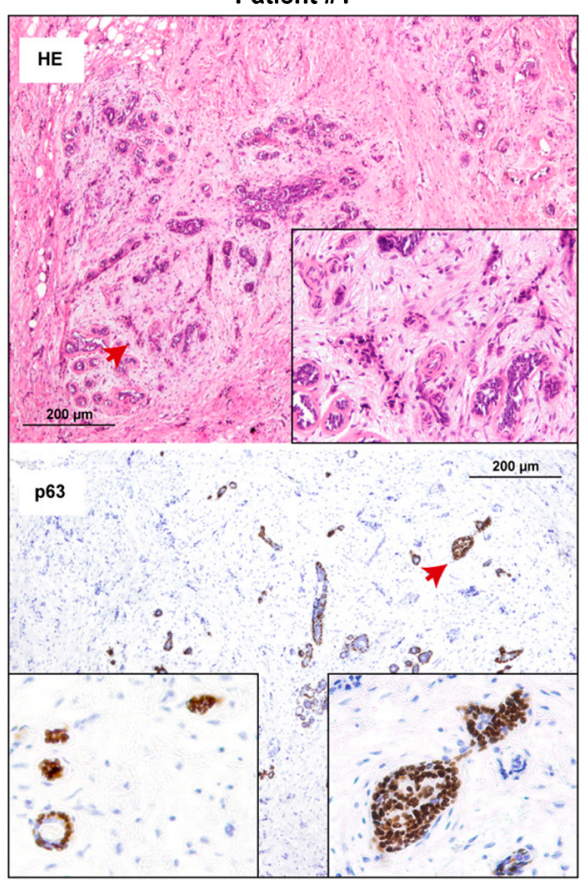

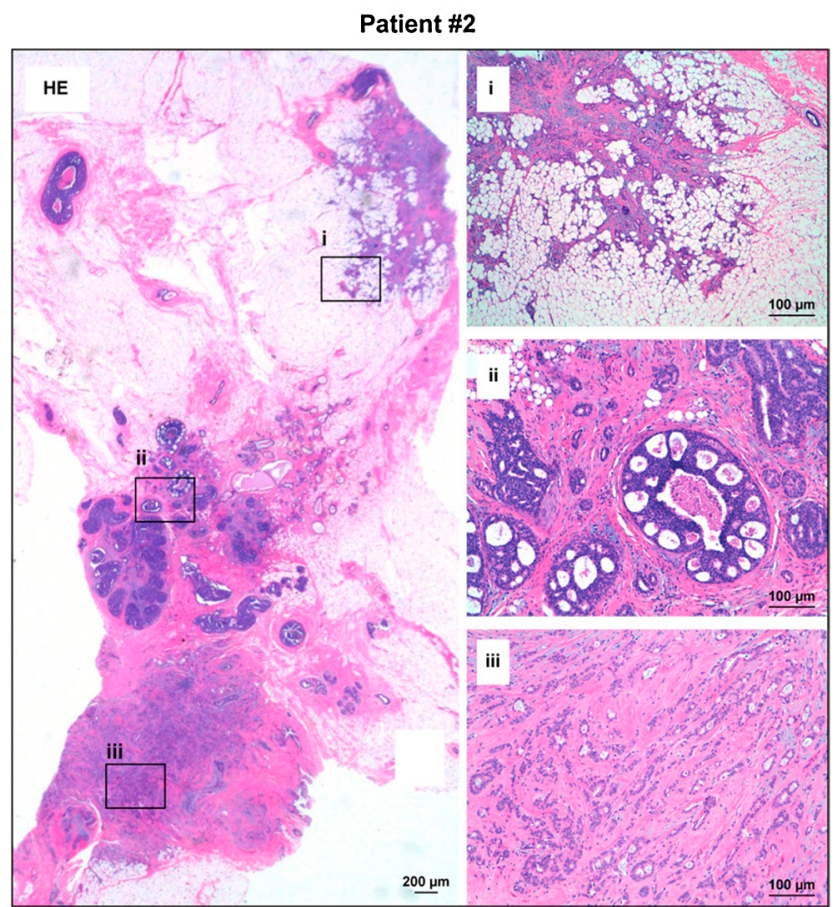

(b)

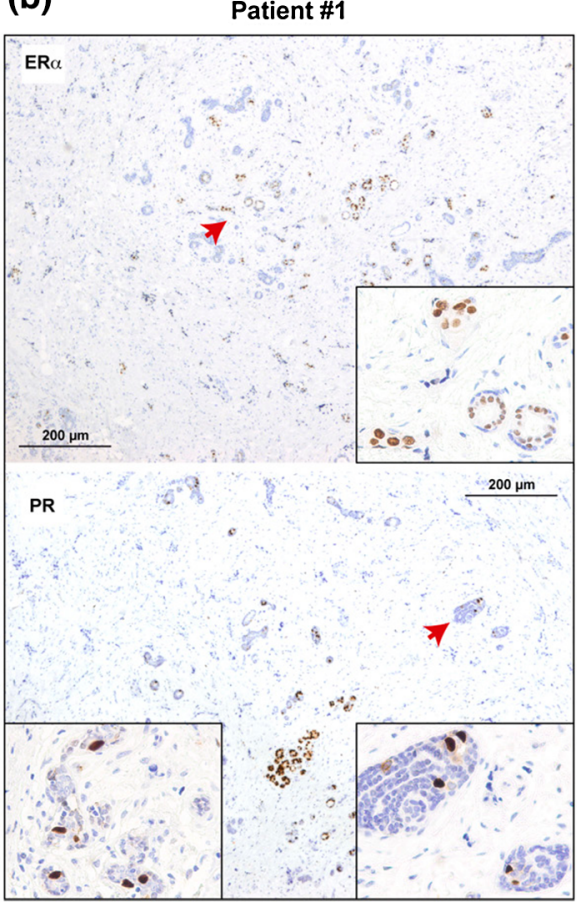

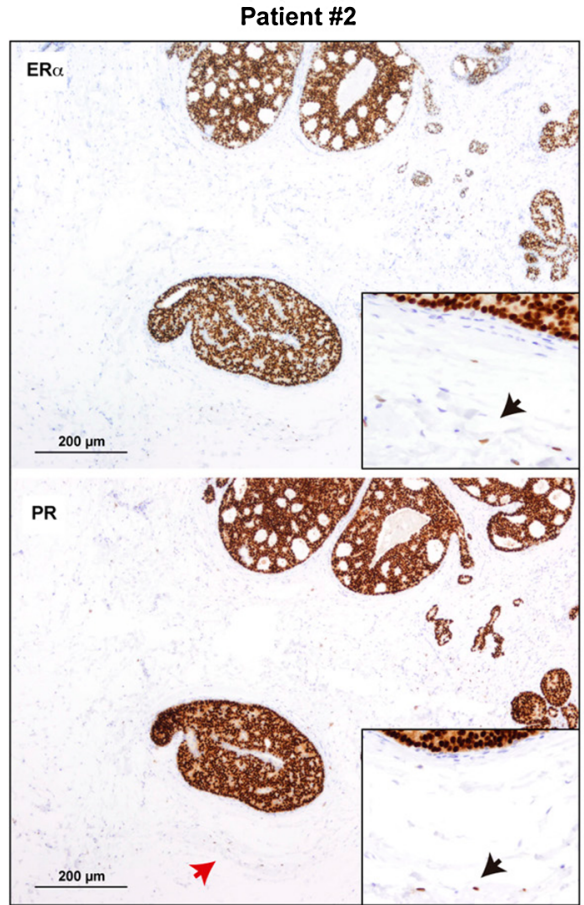

(i) invasive, (ii) in situ, and (iii) desmoplastic histologies. The p63 marker was used as evidence of a metaplastic carcinoma. b Immunostaining results for ER $\alpha$ and PR in the corresponding breast tumors. Red arrows mark magnified fields shown in the insets and black arrows mark positive cells with a spindle-like phenotype

population of ER $\alpha / \mathrm{PR}$-positive cell progenitors [22] leading to an increase in mTORC1 activity, the corresponding cells would possess metastatic behavior with lung tropism. the time window for acquiring somatic genetic mutations by chance. Thus, if a mutation is acquired in a specific of breast tumors in two LAM patients. a Hematoxylin-eosin (HE) and p63 (patient \#1 only) staining results from the corresponding tumors in LAM patients. Arrows mark magnified fields shown in the insets. Three panels are shown for patient \#2, which correspond to

occur in normal breast tissue at reproductive age provide 


\section{(a)}

Patient \#1

Patient \#2

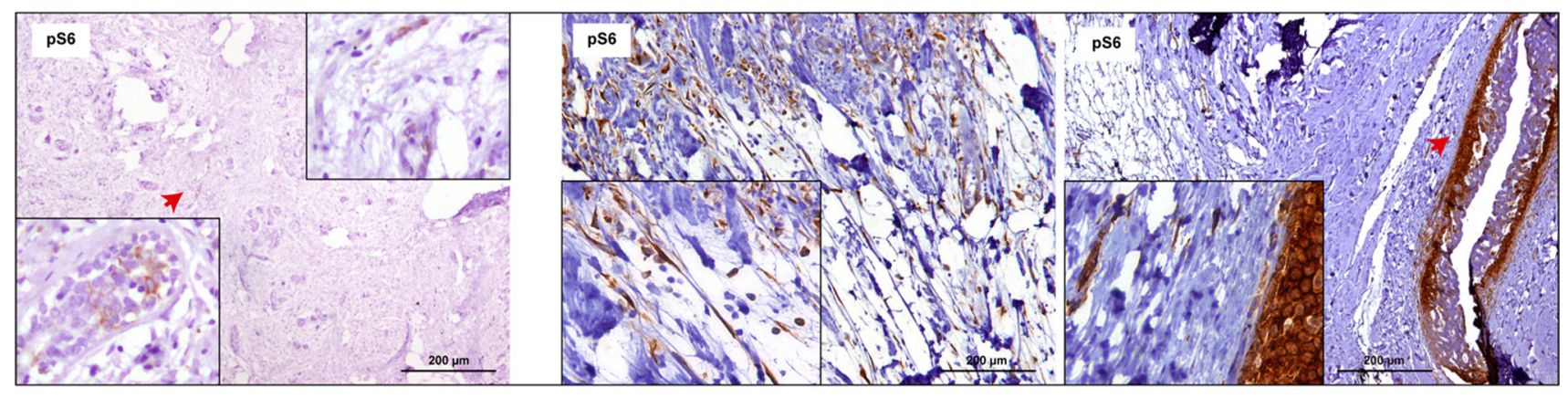

(b)

Patient \#1

Patient \#2

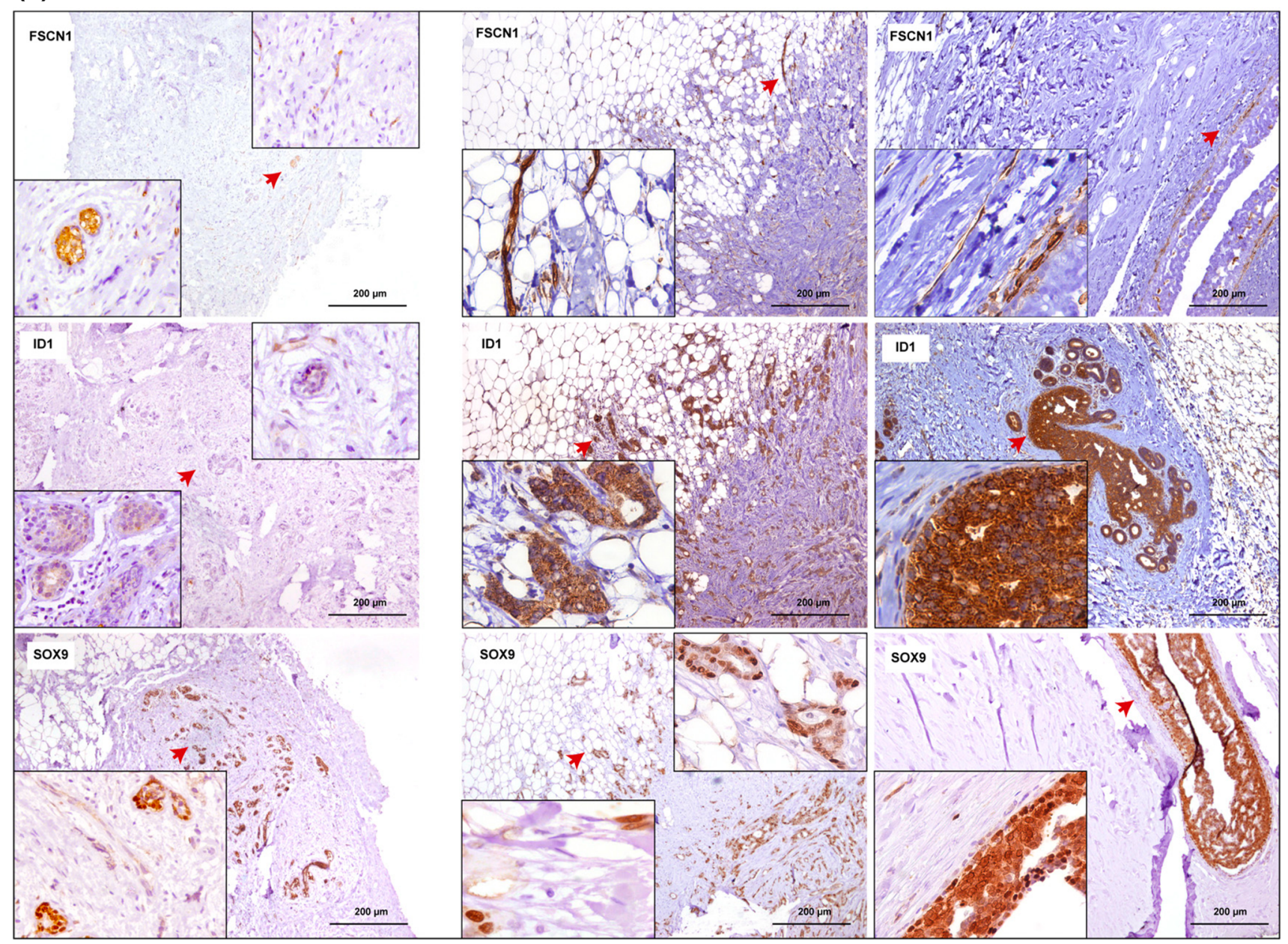

Fig. 2 Positivity for mTORC1 signaling and metastatic markers in breast tumors of LAM patients. a Results of phospho-Ser235/236ribosomal protein S6 (pS6) staining in two available breast tumors. Heterogeneity (i.e., positive and negative tumor cells in case \#1) and positive cells with a spindle phenotype (case \#2, right panels,

Abnormal activation of mTORC1 would not produce invasive tumors unless additional mutations are acquired, which we speculate might have occurred in the identified cases of LAM and breast cancer. Overall, prospective studies may be warranted to corroborate our findings, depicted in insets) can be observed (arrows mark magnified regions). b Results of the analysis of the metastatic markers (FSCN1, ID1, and SOX9) in both cases. Heterogeneity (particularly in case \#1) and spindle-like phenotypes (particularly in case \#2) can be observed (arrows mark magnified regions)

which may be particularly relevant for pre-menopausal women with LAM.

Acknowledgments We wish to thank all patients for their contribution to the work of the corresponding centers, clinics, or 
foundations and owe particular thanks to the Spanish Association of LAM (AELAM) for continuous support during the study. We also wish to thank Judy Sheridan for sharing with us the self-reported United States LAM Foundation data, Prof. Francis McCormack for his valuable comments, and Prof. José Baselga for helping to identify a breast cancer case.

Authors' contributions OM, MP, and MAP conceived of the study and participated in its design. GRG, CH, GM-B, JP, FM, AP, FC, TS, $\mathrm{AV}$, JIL, and NG conducted analysis of pathology specimens. GRG, JVS-M, and ME conducted genetic analyses. AR, SRJ, YI, MH, AC, AG, RO, MJP, MC, EA, MM-M, CV, JA, AX, PU, and RL collected the clinical data. All authors read and approved the final manuscript.

Grant support This work was supported by grants from the “Asociación Española Contra el Cáncer" (AECC, Hereditary Cancer), the Generalitat de Catalunya (SGR 2014-364), the "Instituto de Salud Carlos III" (ISCIII), Spanish Ministry of Economy and Competitiveness, "Fondo Europeo de Desarrollo Regional (FEDER), una manera de hacer Europa" (PIE13/00022-ONCOPROFILE, FIS PI12/ 01528 and PI15/00854, and RTICC RD12/0036/0008), and the Telemaraton 2014 "Todos Somos Raros, Todos Somos Únicos" (P35).

\section{Compliance with ethical standards}

Conflict of interest The authors declare no conflict of interest.

Open Access This article is distributed under the terms of the Creative Commons Attribution-NonCommercial 4.0 International License (http://creativecommons.org/licenses/by-nc/4.0/), which permits any noncommercial use, distribution, and reproduction in any medium, provided you give appropriate credit to the original author(s) and the source, provide a link to the Creative Commons license, and indicate if changes were made.

\section{References}

1. Taveira-DaSilva AM, Moss J (2015) Clinical features, epidemiology, and therapy of lymphangioleiomyomatosis. Clin Epidemiol 7(7):249-257

2. McCormack FX (2008) Lymphangioleiomyomatosis: a clinical update. Chest 133(2):507-516

3. Henske EP, McCormack FX (2012) Lymphangioleiomyomatosis-a wolf in sheep's clothing. J Clin Invest 122(11):3807-3816

4. Zoncu R, Efeyan A, Sabatini DM (2011) mTOR: from growth signal integration to cancer, diabetes and ageing. Nat Rev Mol Cell Biol 12(1):21-35

5. Hsieh AC, Liu Y, Edlind MP, Ingolia NT, Janes MR, Sher A et al (2012) The translational landscape of mTOR signalling steers cancer initiation and metastasis. Nature 485(7396):55-61

6. Jiang WG, Sampson J, Martin TA, Lee-Jones L, Watkins G, Douglas-Jones A et al (2005) Tuberin and hamartin are aberrantly expressed and linked to clinical outcome in human breast cancer: the role of promoter methylation of TSC genes. Eur J Cancer 41(11):1628-1636

7. Nasr Z, Robert F, Porco JA Jr, Muller WJ, Pelletier J (2013) eIF4F suppression in breast cancer affects maintenance and progression. Oncogene 32(7):861-871

8. Ruiz de Garibay G, Herranz C, Llorente A, Boni J, Serra-Musach J, Mateo F et al (2015) Lymphangioleiomyomatosis biomarkers linked to lung metastatic potential and cell stemness. PLoS One 10(7): $\mathrm{e} 0132546$

9. Johnson SR, Cordier JF, Lazor R, Cottin V, Costabel U, Harari S et al (2010) European Respiratory Society guidelines for the diagnosis and management of lymphangioleiomyomatosis. Eur Respir J 35(1):14-26

10. Taveira-DaSilva AM, Moss J (2015) Clinical features, epidemiology, and therapy of lymphangioleiomyomatosis. Clin Epidemiol 7:249-257

11. Breslow NE, Day NE (1987) Statistical methods in cancer research. Volume II- the design and analysis of cohort studies. IARC Sci Publ 82:1-406

12. Kirkwood BR, Sterne JAC (2003) Essential medical statistics. Blackwell Science, Malden

13. Issa A, Gill JW, Heideman MR, Sahin O, Wiemann S, Dey JH et al (2013) Combinatorial targeting of FGF and ErbB receptors blocks growth and metastatic spread of breast cancer models. Breast Cancer Res 15(1):R8

14. Claus EB, Risch NJ, Thompson WD (1990) Using age of onset to distinguish between subforms of breast cancer. Ann Hum Genet 54(Pt 2):169-177

15. Anderson WF, Chatterjee N, Ershler WB, Brawley OW (2002) Estrogen receptor breast cancer phenotypes in the surveillance, epidemiology, and end results database. Breast Cancer Res Treat 76(1):27-36

16. Badri KR, Gao L, Hyjek E, Schuger N, Schuger L, Qin W et al (2013) Exonic mutations of TSC2/TSC1 are common but not seen in all sporadic pulmonary lymphangioleiomyomatosis. Am J Respir Crit Care Med 187(6):663-665

17. TCGA (2012) Comprehensive molecular portraits of human breast tumours. Nature 490(7418):61-70

18. McCormack FX, Travis WD, Colby TV, Henske EP, Moss J (2012) Lymphangioleiomyomatosis: calling it what it is: a lowgrade, destructive, metastasizing neoplasm. Am J Respir Crit Care Med 186(12):1210-1212

19. Delaney SP, Julian LM, Stanford WL (2014) The neural crest lineage as a driver of disease heterogeneity in tuberous sclerosis complex and lymphangioleiomyomatosis. Front Cell Dev Biol 2:69

20. Al-Saleem T, Wessner LL, Scheithauer BW, Patterson K, Roach ES, Dreyer SJ et al (1998) Malignant tumors of the kidney, brain, and soft tissues in children and young adults with the tuberous sclerosis complex. Cancer 83(10):2208-2216

21. Meric-Bernstam F, Brusco L, Daniels M, Wathoo C, Bailey A, Strong L et al (2016) Incidental germline variants in 1000 advanced cancers on a prospective somatic genomic profiling protocol. Ann Oncol. doi:10.1093/annonc/mdw018

22. Fu N, Lindeman GJ, Visvader JE (2014) The mammary stem cell hierarchy. Curr Top Dev Biol 107:133-160 